\title{
Prohibitin S-Nitrosylation Is Required for the Neuroprotective Effect of Nitric Oxide in Neuronal Cultures
}

\author{
Youyang Qu, ${ }^{1,2 *}$ Csaba Konrad, ${ }^{1 *}$ Corey Anderson, ${ }^{1}$ Liping Qian, ${ }^{1}$ Tina Yin, ${ }^{1}$ Giovanni Manfredi, ${ }^{1}$ \\ Costantino Iadecola, ${ }^{1}$ and Ping Zhou ${ }^{1}$ \\ ${ }^{1}$ Feil Family Brain and Mind Research Institute, Weill Cornell Medicine, New York, New York 10065, and ${ }^{2}$ Department of Neurology, 2nd Affiliated \\ Hospital of Harbin Medical University, Harbin 150086, People's Republic of China
}

Prohibitin (PHB) is a critical protein involved in many cellular activities. In brain, PHB resides in mitochondria, where it forms a large protein complex with PHB2 in the inner TFmembrane, which serves as a scaffolding platform for proteins involved in mitochondrial structural and functional integrity. PHB overexpression at moderate levels provides neuroprotection in experimental brain injury models. In addition, PHB expression is involved in ischemic preconditioning, as its expression is enhanced in preconditioning paradigms. However, the mechanisms of PHB functional regulation are still unknown. Observations that nitric oxide (NO) plays a key role in ischemia preconditioning compelled us to postulate that the neuroprotective effect of PHB could be regulated by NO. Here, we test this hypothesis in a neuronal model of ischemia-reperfusion injury and show that $\mathrm{NO}$ and $\mathrm{PHB}$ are mutually required for neuronal resilience against oxygen and glucose deprivation stress. Further, we demonstrate that NO post-translationally modifies PHB through protein S-nitrosylation and regulates PHB neuroprotective function, in a nitric oxide synthase-dependent manner. These results uncover the mechanisms of a previously unrecognized form of molecular regulation of PHB that underlies its neuroprotective function.

Key words: neuroprotection; nitric oxide; prohibitin; protein S-nitrosylation

Significance Statement

Prohibitin (PHB) is a critical mitochondrial protein that exerts a potent neuroprotective effect when mildly upregulated in mice. However, how the neuroprotective function of PHB is regulated is still unknown. Here, we demonstrate a novel regulatory mechanism for PHB that involves nitric oxide (NO) and shows that PHB and NO interact directly, resulting in protein Snitrosylation on residue Cys ${ }^{69}$ of PHB. We further show that nitrosylation of PHB may be essential for its ability to preserve neuronal viability under hypoxic stress. Thus, our study reveals a previously unknown mechanism of functional regulation of PHB that has potential therapeutic implications for neurologic disorders.

\section{Introduction}

Prohibitin (PHB) also known as prohibitin1 (PHB1, herein $\mathrm{PHB})$ is a nuclear encoded protein that localizes in brain mitochondria (Zhou et al., 2012) and is essential for life, as germline PHB gene deletion leads to embryonic death in Caenorhabditis elegans (Artal-Sanz et al., 2003) and in mice (Park et al., 2005). $\mathrm{PHB}$ resides in the inner mitochondrial membrane where it forms a high-molecular weight complex with its close family member, PHB2, to serve as a scaffold essential for several

\footnotetext{
Received July 26, 2019; revised Feb. 25, 2020; accepted Feb. 26, 2020.

Author contributions: G.M., C.I., and P.Z. designed research; Y.Q., C.K., C.A., L.Q., and T.Y. performed research; Y.Q., L.Q., and P.Z. analyzed data; P.Z. wrote the paper.

*Y.Q. and C.K. contributed equally to this work.

The authors declare no competing financial interests.

This work was supported in part by National Institutes of Health Grants R01-NS-067078 (to P.Z.) and R01NS-034179 (to C.I.).

Correspondence should be addressed to Ping Zhou at piz2001@med.cornell.edu.

https://doi.org/10.1523/JNEUROSCl.1804-19.2020

Copyright $\odot 2020$ the authors
}

mitochondrial functions (Merkwirth and Langer, 2009; Thuaud et al., 2013). PHB is involved in the maintenance of mitochondrial morphology (Steglich et al., 1999; Nijtmans et al., 2000; Merkwirth et al., 2008; Anderson et al., 2018), dynamics regulation (Merkwirth et al., 2008), and respiratory chain complex assembly and stability (Anderson et al., 2018, 2019). Studies from us and others have demonstrated that PHB expression is beneficial in counteracting the oxidative stress caused by glutamate in neurons (Zhou et al., 2012) and the inflammatory response-associated oxidative stress and injury in mouse intestinal epithelium (Theiss et al., 2009). When expressed even at modest levels in mice, $\mathrm{PHB}$ exhibits a robust neuroprotective effect against ischemic brain injury (Kahl et al., 2018). However, despite these functional characterizations, little is known about how this important protein is functionally regulated.

Nitric oxide (NO) is an important signaling molecule. Recent studies showed that $\mathrm{NO}$ is involved in the development of ischemic tolerance, also termed ischemic preconditioning (IPC), a phenomenon in which a short-term, mild ischemic insult to any 
organ induces the development of substantial resistance to a subsequent major ischemic attack (Murry et al., 1986). Involvement of NO produced by all three isoforms of NO synthase (NOS) in IPC signaling has been substantiated in numerous studies. Inducible NOS-derived NO plays an obligatory role in ischemic preconditioning in mouse brain (Cho et al., 2005). Moreover, genetic deletion of either neuronal NOS (nNOS) or endothelial NOS renders mice insensitive to IPC stimulation and abolishes IPC-mediated neuroprotection, demonstrating an essential role of NO in the protective effect of IPC (Atochin et al., 2003). Together, these studies underscore the importance of NO in IPC. However, the mechanisms and the targets involved in NO-mediated neuroprotection remain elusive.

Based on the requirement of NO for IPC induction, IPC upregulation of $\mathrm{PHB}$ expression (Zhou et al., 2012), and the neuroprotection afforded by $\mathrm{PHB}$ expression, we hypothesized the existence of a mechanistic link between NO and PHB in neuroprotection. Here, we test this hypothesis using oxygen and glucose deprivation (OGD), an in vitro neuronal model of ischemic stress. We show that $\mathrm{NO}$ and $\mathrm{PHB}$ are mutually required to maintain cell viability in hypoxic stress, and that mechanistically $\mathrm{NO}$ directly interacts with $\mathrm{PHB}$, resulting in $\mathrm{PHB}$ protein Snitrosylation in its sole Cys residue. Furthermore, we show that endogenous NO formation during neural activity is able to modulate the protective effect of $\mathrm{PHB}$ via nitrosylation.

\section{Materials and Methods}

\section{Materials}

Tissue culture reagents (medium, serum, salt supplements, and solutions) were purchased from Thermo Fisher Scientific. Protein S-nitrosylation reagents ( $N$-[6-(biotinamido)hexyl]-3'-(2'-pyridyldithio) propionamide and streptavidin-agarose beads) were from Thermo Fisher Scientific, and NO donors were from Cayman Chemicals. Other chemicals were from Sigma-Aldrich. Anti-PHB antibodies were from Thermo Fisher Scientific. Precast SDS polyacrylamide gels were from Bio-Rad.

\section{Primary cortical neuronal culture}

The experimental procedures for primary neuronal culture using timed pregnant mice were approved by the Institutional Animal Care and Use Committee of Weill Cornell Medicine. Primary neuronal cultures were prepared following an established procedure, as described previously (Zhou et al., 2012). Briefly, cortices from day 16 embryos (C57BL/6) were dissected out into digestion buffer containing $0.25 \%$ trypsin and $0.15 \mu \mathrm{g} /$ $\mathrm{ml} \mathrm{DNase} \mathrm{I.} \mathrm{The} \mathrm{tissue} \mathrm{was} \mathrm{incubated} \mathrm{at} 37^{\circ} \mathrm{C}$ for $4 \mathrm{~min}$ followed by triturating using a fire-polished glass Pasteur pipette. The dissociated cells were diluted into $10 \mathrm{ml}$ of HBSS plus $10 \%$ FBS. The suspension was collected and centrifuged. The pellet was resuspended into Neurobasal medium supplemented with $2 \%$ B27. The cells were counted and seeded onto polyD-lysine-coated plates at a density of $8 \times 105 / \mathrm{ml}$ for incubation at $37^{\circ} \mathrm{C}$. The medium was changed twice a week, and the cultures were maintained for 5 or $12 \mathrm{~d}$, depending on the purpose of the experiments.

\section{siRNA transfection into primary neurons}

siRNA specific for mouse PHB (si-PHB, GGGACUCAUUUCCUCAUCCtt; Thermo Fisher Scientific) was introduced into primary neurons by using Lipofectamine 3000 (Thermo Fisher Scientific) following the protocol from manufacturer. siRNA with scrambled sequences that do not have a homolog to known mammalian sequence was used to serve as a negative control. Primary neurons were plated into six-well plates or $15 \mathrm{~cm}$ dishes, transfected with siRNA on day 7, and cultured for 5 more days before being used for experiments.

\section{Oxygen and glucose deprivation and cell death assessment}

Injury in neuronal culture was produced by OGD, as described previously (Zhou et al., 2012). To start OGD, medium in neuronal cultures
(12 days in culture) was replaced with buffered salt solution without glucose and cells were subjected to OGD for $4 \mathrm{~h}$ in a hypoxia chamber (Billups-Rothenberg) by flushing the chamber with $95 \% \mathrm{~N}_{2}$ and $5 \%$ $\mathrm{CO}_{2}$. Sham cultures were treated with buffer containing glucose under normoxia and served as controls. After OGD treatment, cells were washed, and normal culture medium was added and returned to the incubator for an additional $24 \mathrm{~h}$ before cell viability assessment by morphologic criteria as described previously (Zhou et al., 2005) with modifications. For nuclear morphology assessment, cells were first fixed in $4 \%$ paraformaldehyde for $10 \mathrm{~min}$ followed by incubation in PBST (PBS buffer with $0.1 \%$ Triton X-100) for $10 \mathrm{~min}$. The cells were then incubated with DAPI $(0.5 \mu \mathrm{g} / \mathrm{ml}$ in PBS $)$ for $10 \mathrm{~min}$ at room temperature. The cells were then imaged under a fluorescence microscope equipped with a DAPI filter (Nikon). Both DAPI and corresponding bright-field images (BFIs) from 10-15 randomly selected fields were taken at $20 \times(50-100$ cells/field) for each plate of cells. Merged DAPI images and BFIs of the same field were used to identify neurons and exclude them from counting cell debris and astrocytes. Live or dead cells were determined based on nuclei morphology in DAPI images and cell body-associated neurite intactness in BFI. Cells with fragmented or condensed nuclei and fragmented neurites in continuity with the cell body were counted as dead, while cells with uncondensed nuclei and intact cell bodies and associated neurites were considered alive. In case there were cells with intact nuclei but fragmented neurites, they were excluded from counting either as alive or dead. In some experiments as indicated, cell viability was also assayed by MTS cellular metabolism measurement with reagents from Promega.

\section{Quantitative real-time PCR analysis of mouse PHB expression in neuronal cultures}

PHB mRNA levels after DPTA (diethylenetriaminepentaacetic acid) stimulation were analyzed by quantitative PCR (qPCR), as described previously (Kahl et al., 2018). Briefly, total RNAs from primary neurons were extracted by Invitrogen TRIzol (Thermo Fisher Scientific) according to the manufacturer instructions. After cDNA synthesis from total RNA $(5 \mu \mathrm{g})$, qPCR was performed by SYBR Premix EX Taq in a Bio-Rad IQ5 PCR System. PCR was performed under the following conditions: denaturation at $95^{\circ} \mathrm{C}$ for $2 \mathrm{~min}, 40$ cycles of denaturation at $95^{\circ} \mathrm{C}$ for 15 $\mathrm{s}$, annealing at $60^{\circ} \mathrm{C}$ for $20 \mathrm{~s}$, and extension at $72^{\circ} \mathrm{C}$ for $20 \mathrm{~s}$, followed by a final extension at $72^{\circ} \mathrm{C}$ for $5 \mathrm{~min}$. Finally, the melting curve analysis was performed to confirm that a single product was amplified without primer dimers interfering with the reaction. The comparative $\mathrm{Ct}$ method (2- $\Delta \Delta \mathrm{Ct})$ was used for the relative mRNA quantification.

\section{Protein nitrosylation assay}

A biotin switch assay (Jaffrey et al., 2001) was used to detect the protein Cys-S nitrosylation status of PHB. The assay was performed according to the standard procedure (Forrester et al., 2009). Briefly, lysates of neuronal culture or brain tissue were prepared in HEN buffer $(100 \mathrm{~mm}$ HEPES, 1 mM EDTA, $0.1 \mathrm{~mm}$ neocuproine, $\mathrm{pH} 8.0$ ). After protein concentration determination, $0.6 \mathrm{mg}$ of protein $(1.8 \mathrm{ml})$ were aliquoted into each tube, blocking reagents added [final concentration: $2.5 \%$ SDS, $0.1 \%$ methyl methane thiosulfonate (MMTS)], and incubated for $20 \mathrm{~min}$ at $50^{\circ} \mathrm{C}$ to block free-thiol groups. Excess MMTS was removed by cold acetone precipitation (three volumes) followed by $70 \%$ acetone washes. The pellets were resuspended in HENS buffer (HEN with 1\% SDS) and the free nitrosothiols were reduced to thiols with ascorbate $(20 \mathrm{mM})$ in the presence of $N$-[6-(biotinamido)hexyl] $-3^{\prime}-\left(2^{\prime}\right.$-pyridyldithio)propionamide (biotin-HPDP; $2.5 \mathrm{mg} / \mathrm{ml}$ ). An aliquot of the samples was saved before the next step to serve as a loading control (input) in the detection step. Biotinylated proteins were pulled down with streptavidin-agarose beads, and the pellet was washed four times with wash buffer $(25 \mathrm{~mm}$ HEPES, pH 7.5; 100 mm NaCl; 1 mм EDTA; 0.5\% Triton X-100; 600 mm $\mathrm{NaCl}$ ). The proteins on beads were then eluted by incubating with elution buffer (10 mM HEPES, pH 8.0; 0.1 mM EDTA; $1 \% \beta$-mercaptoethanol), followed by a quick spin for $30 \mathrm{~s}$. The supernatants were collected, mixed with SDS-PAGE sample loading buffer, and loaded onto $12 \%$ polyacrylamide gels (Bio-Rad). The nitrosylated proteins were detected by Western blot analysis. 

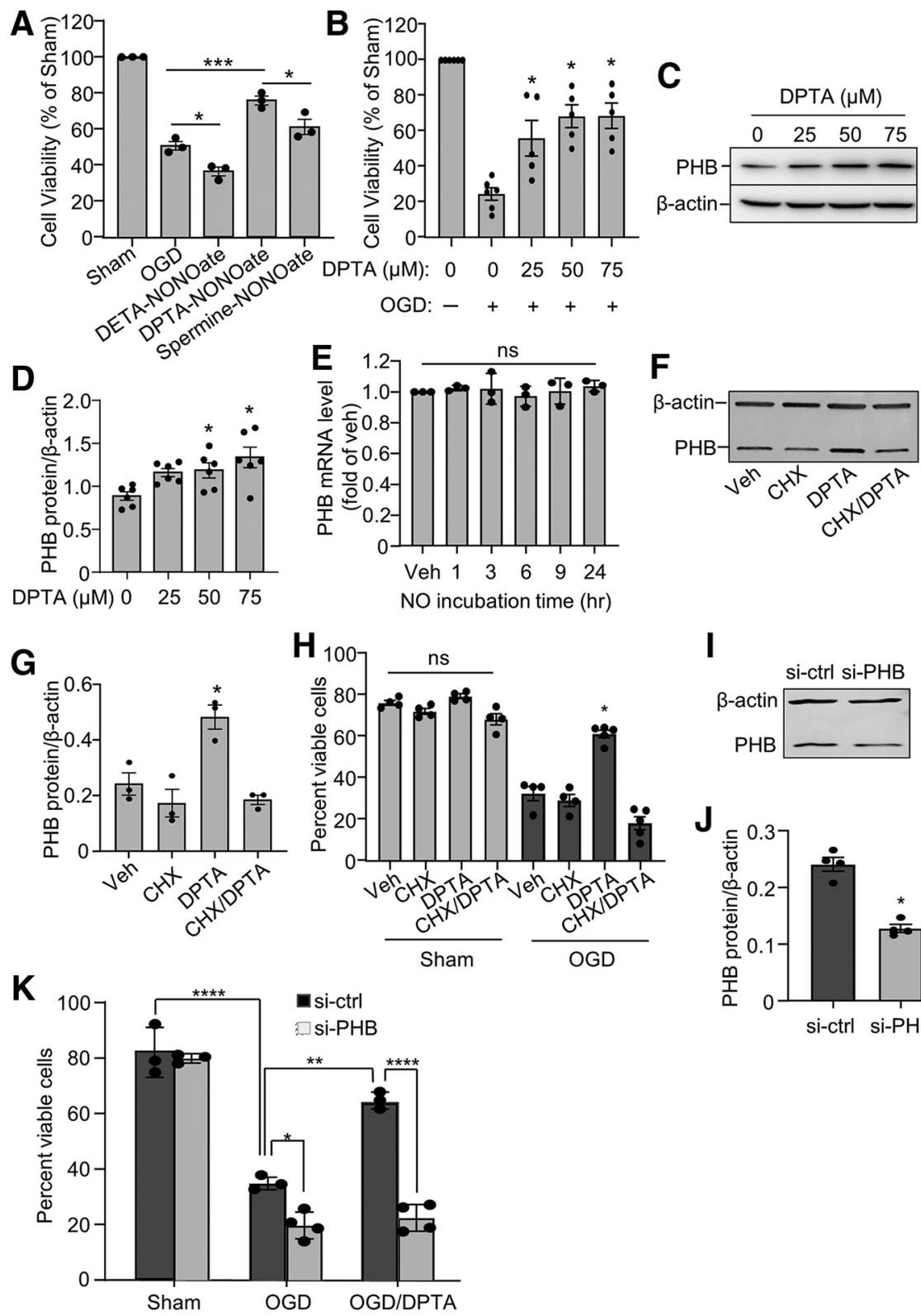

$\mathbf{F}$
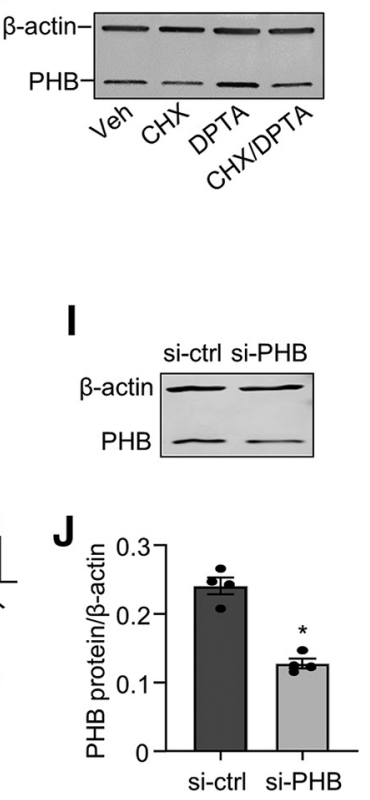

Figure 1. Characterization of a model of N0-mediated neuroprotection. All experiments were conducted with DIV14 (days in vitro) primary neurons treated with indicated N0 donor for $12 \mathrm{~h}$ followed by assessment of cell viability or PHB protein levels. $A, N 0$ donor screening for their ability to protect neurons against OGD-induced cell death. Cells were treated with three N0N0atebased NO donors $(50 \mu \mathrm{m})$ for $12 \mathrm{~h}$ followed by OGD for $4 \mathrm{~h}$. Twenty-four hours after OGD, cell viability was assessed and expressed as a percentage of sham controls. ${ }^{* * *} p<0.001,{ }^{*} p<0.05, n=3 /$ group. $\boldsymbol{B}$, After DPTA treatment, cells were subjected to OGD for $4 \mathrm{~h}$ followed by $20 \mathrm{~h}$ of culture in normal conditions. Cell viability was then analyzed based on morphologic criteria (for details, see Methods) and presented as a percentage of controls. ${ }^{*} p<0.05$ compared with untreated 0GD samples, $n=3$ separate experiments with duplicate set of samples. C, After DPTA incubation, cell lysates were made for Western blot analysis of PHB protein levels. $\beta$-Actin was used as a loading control. D, PHB protein band quantification. Band intensity of PHB was normalized to $\beta$-actin and expressed in arbitrary units; ${ }^{*} p<0.05, n=3$ separate experiments with a duplicate set of samples. $\boldsymbol{E}, \mathrm{qPCR}$ measurement of PHB mRNA in N0 donor-treated neurons. Neuronal cultures were incubated with DPTA $(50 \mu \mathrm{M})$, and cells were lysed for RNA isolation at the indicated time. The relative changes of mouse PHB mRNA in neurons after N0 stimulation was quantitated by the $2^{-\Delta \Delta \sigma}$ method from SYBR Green amplified PCR products. Results are normalized to the vehicle-treated cells and are expressed as fold changes. Three separated experiments were performed. ns, Not significant. $\boldsymbol{F}$, NO donor-induced PHB protein increase is blocked by CHX. Neurons were preincubated with CHX (100 $\mu \mathrm{m}, 2 \mathrm{~h})$ before DPTA $(50 \mu \mathrm{m})$ was added. After $6 \mathrm{~h}$ of coincubation, cells were lysed for PHB protein analysis. Shown is one representative Western blot image from three independent experiments. Each lane was loaded with $15 \mu \mathrm{g}$ of lysate. $\beta$-Actin was used as loading control. G, PHB protein band intensity quantification normalized to loading control and presented as arbitrary units. ${ }^{*} p<0.01, n=3$ separate experiments. $\boldsymbol{H}$, Cell viability assessment in neurons after CHX/DPTA coincubation and OGD treatment. Neurons were treated as indicated for $6 \mathrm{~h}$ followed by OGD for $4 \mathrm{~h}$. Cell viability was assessed after $20 \mathrm{~h}$ by morphologic criteria. ${ }^{*} p<0.01$, $n=4$ experiments with $>250$ cells per experiment in each group. ns, not significant. $I$, Western blot demonstrating PHB downregulation in parallel neuronal cultures $5 \mathrm{~d}$ after transfection. J, PHB protein band intensity measurement after knockdown. Data

\section{Western blot}

After protein concentration determination with DC reagents (Bio-Rad), equal amounts of proteins were loaded on premade $12 \%$ polyacrylamide gel (Bio-Rad), separated, and transferred to PVDF membranes. Membranes were incubated with specific antibodies in appropriate dilutions after blocking with 5\% dry milk in PBS for $1 \mathrm{~h}$ at room temperature. The membranes were washed three times with PBST (PBS $+0.1 \%$ Tween-20), incubated with appropriate secondary antibodies for $1 \mathrm{~h}$, and washed, and images were acquired with a CLx Imaging Station (LI-COR). Band intensity was analyzed using Image Studio software (version 3.1; LI-COR).

PHB protein sequence alignment analysis Amino acid sequences of PHB1 and PHB2 for all species from human to fly and fungus were obtained from the GenBank database. Sequences were aligned using the Clustal Omega program from UniProt (www.uniprot. org/align).

cGMP measurement in neuronal culture

The cGMP content in neuronal cells was measured with a Parameter cGMP assay kit (R\&D Systems) following manufacturer protocols. Briefly, cells from different treatments were washed, lysis buffer was added, and cells were frozen-thawed two times. After a brief spin to remove cell debris, the cell lysates were collected for assay. The lysate samples were added to the assay plate and incubated with HRP-conjugated cGMP in fixed amount and an antibody against cGMP for $3 \mathrm{~h}$. After four washes, color developing reagents were added to the plate and the absorbance was read at $450 \mathrm{~nm}$. The cGMP content was derived by calculating from a standard curve constructed with a serial dilution of known concentration of cGMP.

\section{NO measurement with fluorescent} indicator DAF-FM

NO generated from nNOS on NMDA receptor activation was detected using the fluorescent NO indicator Invitrogen DAF-FM diacetate (5 mM DMSO stock; catalog \# D-23 844, Thermo Fisher Scientific). Neurons [day in vitro 14 (DIV14)] were pretreated with L-NAME (100 $\mu \mathrm{M})$ for $2 \mathrm{~h}$ followed by bicuculline (Bic; $50 \mu \mathrm{M}$ ) for $1 \mathrm{~h}$. Cells were washed with warm medium twice and then incubated with DAF-FM for $30 \mathrm{~min}$. After washing three times with PBS to remove any extracellular dye, the cells were

$\leftarrow$

were normalized to loading controls and expressed in arbitrary units. ${ }^{*} p<0.05, n=4$ sets of samples from three separate experiments. $\boldsymbol{K}, \mathrm{PHB}$ expression is required for the protective effect of NO in neuronal OGD. Neurons at DIV7 were transfected with mouse-specific siRNA (si-PHB) and control siRNA (si-ctrl) and cultured for 5 more days. Cultures were then treated with DPTA $(50 \mu \mathrm{m})$ followed by $O G D$ and viability assessment. Data show that NO fails to improve neuronal viability when endogenous $\mathrm{PHB}$ is downregulated by siRNA. ${ }^{*} p<0.05,{ }^{* *} p<0.01,{ }^{* * * *} p<0.001, n=3$ separate experiments. ns, Not significant. 
A

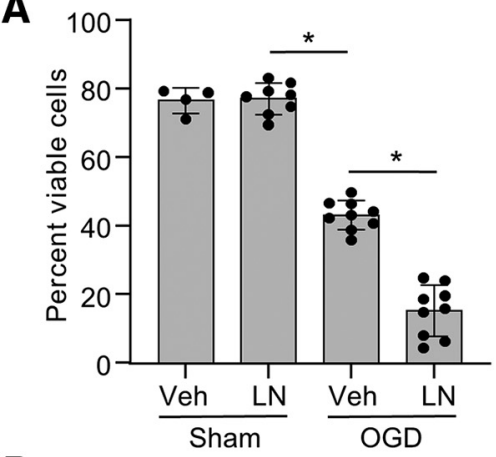

D

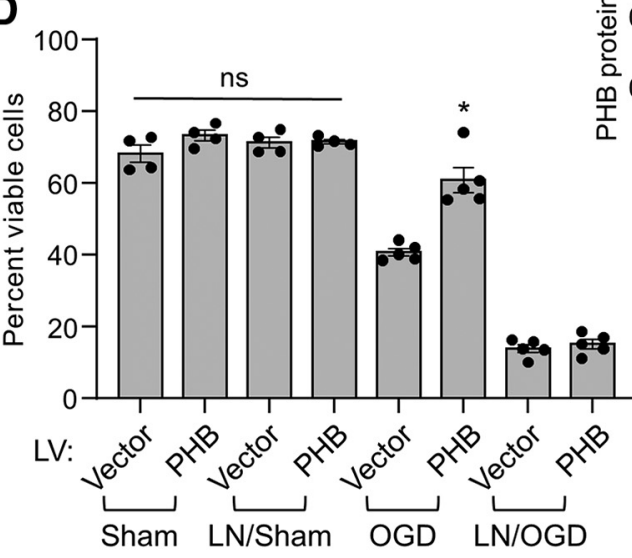

E

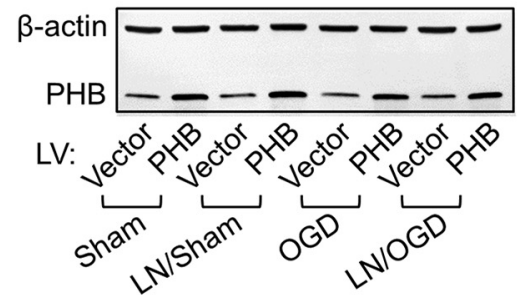

B

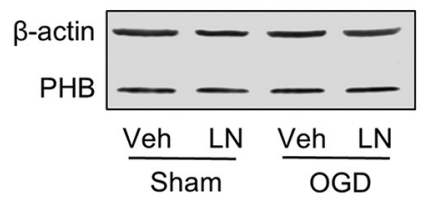

C

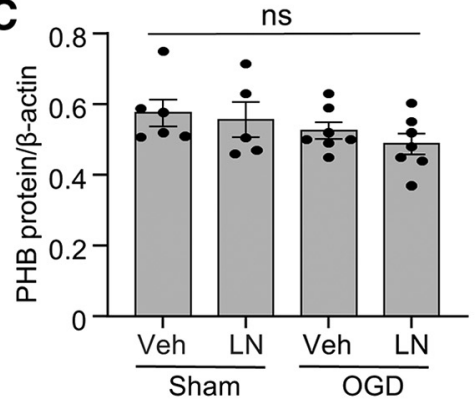

was used as a positive control, respectively. In siRNA-mediated PHB knockdown, a scrambled sequence that had no effect on cell viability and PHB expression was used as control. We performed a neuronal cell viability assay based on cell morphology changes after OGD. To avoid bias, the cell plates being assayed were coded and the identities of the cells were kept blind for designated personnel in image analyses. At least 10 randomly selected fields for each cell type were imaged, and at least 200 cells per cell type were analyzed. Three to five independent experiments were performed. For statistical analyses, all data are presented as the mean \pm SEM. Statistical differences between two groups were evaluated by unpaired, two-tailed $t$ test. ANOVA with Tukey's post hoc test was used to compare differences across multiple groups. Differences were considered significant at $p<0.05$.

\section{Results}

Increased PHB protein level is a prerequisite in NO-mediated maintenance of cell viability in neurons exposed to OGD

To establish an in vitro model of NO-mediated neuroprotection, we first screened several NO donors with different half-lives for their ability to preserve cortical mouse neurons viability under conditions of OGD. Among the NO donors tested, DPTANONOate (DPTA) displayed the strongest neuroprotection (Fig. 1A). Therefore, in this study we used DPTA as an NO donor. Taking advantage of this NO-stimulated neuroprotection model, we tested whether NO released from DPTA was able to enhance cell viability in association with increased PHB protein levels in neurons exposed to OGD. We incubated neuronal cultures with DPTA at different concentrations and assayed cell viability and $\mathrm{PHB}$ protein levels. We found that DPTA resulted in a significant decrease of OGDinduced cell death, in a dose-dependent manner (Fig. 1B). In addition, DPTA increased neuronal $\mathrm{PHB}$ protein content (Fig. 1C,D), mimicking the effect of IPC in vivo (Zhou et al., 2012). Interestingly, quantitative real-time PCR with mRNA samples from a set of DPTA-treated samples showed that $\mathrm{PHB}$ transcription was unchanged within the time frame of NO-induced $\mathrm{PHB}$ protein increase (Fig. imaged with an ImageXpress Pico Automated Cell Imaging System (Molecular Devices). Fluorescence intensity of the cells was quantitated with ImageJ software after the subtraction of background fluorescence in cells without the dye.

Experimental design and statistical analysis

All the experiments were designed with proper controls. The NO donor experiments were vehicle controlled. Neuronal viability/OGD experiments were sham controlled. In a biotin switch assay, ascorbic acid omission, or NOS inhibitor, was used as a negative control and a NO donor

$1 E)$, suggesting that $\mathrm{NO}$-induced $\mathrm{PHB}$ upregulation does not occur at the transcriptional level. Together, these data demonstrate that donor-derived $\mathrm{NO}$ is able to raise $\mathrm{PHB}$ protein levels and provide neuroprotection against OGD.

To understand how NO induces $\mathrm{PHB}$ protein increase without enhancing transcription, we preincubated neurons with the protein synthesis inhibitor cycloheximide (CHX; $100 \mu \mathrm{M})$ for $2 \mathrm{~h}$ 
preceding DPTA addition, followed by OGD treatment and cell viability assessment. As expected, DPTA-induced PHB protein increase was completely blocked by CHX (Fig. 1F,G), suggesting that $\mathrm{NO}$-mediated $\mathrm{PHB}$ upregulation occurs at the protein synthesis level. Furthermore, when cells were incubated with $\mathrm{CHX}$ first, followed by DPTA, NO-mediated neuroprotection was abolished (Fig. $1 H$ ), suggesting that protein synthesis is needed for NO-mediated neuroprotection.

To specifically demonstrate the role of $\mathrm{PHB}$ upregulation in NO-mediated neuroprotection, we downregulated PHB expression with siRNA before exposing neurons to NO and OGD. Transfection of PHB-specific siRNA for $5 \mathrm{~d}$ reduced endogenous PHB to $\sim 50 \%$ of a scrambled si-control (si-ctrl; Fig. 1I,J), but did not affect neuronal viability in normal conditions (Fig. 1K), consistent with our previous study (Zhou et al., 2012). Conversely, neurons with reduced $\mathrm{PHB}$ were more sensitive to OGD compared with neurons treated with scrambled control siRNA (Fig. 1K). Strikingly, the neuroprotective effect of DPTA was completely abolished by PHB downregulation. These results demonstrate that normal PHB protein levels are required for NO-mediated neuroprotection and that PHB participates in the neuroprotective mechanisms downstream of NO signaling.

\section{NO is essential for PHB expression-mediated neuroprotection}

Having established that PHB is required for NOmediated neuroprotection, we next investigated the role of $\mathrm{NO}$ in PHB-mediated neuroprotection. We used L-NAME, a nonselective NOS inhibitor, to deplete NO in neurons followed by hypoxic stress. In neuronal cultures without OGD, NO depletion did not affect cell viability (Fig. $2 A$ ), indicating that acute NO depletion in normal conditions is not harmful to cells and that no toxic effects of $\mathrm{L}-$ NAME were apparent at the dose used. However, L-NAME treatment resulted in greater neuronal death than vehicle in OGD. The increased cell death was not attributable to altered PHB protein levels, as PHB levels were unchanged in all treatment groups (Fig. 2B,C). These results demonstrate a critical role of $\mathrm{NO}$ in PHB neuroprotection, independent of PHB levels.

To further demonstrate that the effects of NO depletion on neuronal survival in OGD are dependent on PHB, we overexpressed PHB by lentivirus (LV) transduction. As expected, without OGD, NO depletion did not cause a decline in cell viability (Fig. 2D, Sham panel), and, when cells were exposed to OGD without LNAME, overexpression of PHB with LV-PHB transduction provided neuroprotection (Fig. 2D). Strikingly, L-NAME completely abolished the neuroprotection afforded by LV-PHB transduction, without affecting protein overexpression (Fig. 2E,F). These results clearly demonstrate the role of $\mathrm{NO}$ for $\mathrm{PHB}$ protective function, as increasing PHB protein levels in the absence of NO is insufficient for neuroprotection. Thus, $\mathrm{NO}$ is necessary for the protective effect of PHB and, conversely, endogenous PHB content is likely required for $\mathrm{NO}$ preconditioning.
NO effects on PHB neuroprotection are not mediated by the soluble guanylyl cyclase/cGMP pathway

Next, we sought to understand how NO modulates PHB function in neuroprotection. $\mathrm{NO}$ was originally identified as a vasodilating factor from endothelium through activation of guanylyl cyclase to produce the second messenger cGMP (Murad, 1986; Palmer et al., 1987; Russwurm and Koesling, 2004). More recently, an alternative NO signaling pathway has been discovered in which $\mathrm{NO}$ directly reacts with the free thiol group $(-\mathrm{SH})$ of amino acid cysteine to form S-nitrosocysteine, a post-translational protein modification process termed protein S-nitrosylation (Hess et al., 2005; Nakamura and Lipton, 2016; Stomberski et al., 2019). To delineate which pathway is involved in NO modulation of PHB, we first assayed cGMP production in response to DPTA. As anticipated, we observed a time-dependent increase of cellular cGMP with DPTA (Fig. $3 A$ ), demonstrating that the cGC/cGMP system is functional in our experimental model. We then used ODQ, a potent cGC inhibitor (Garthwaite et al., 1995), to investigate the role of cGMP on the protective effect of DPTA. ODQ $(10 \mu \mathrm{M})$ blocked the increase in cGMP induced by DPTA (Fig. $3 B$ ) without altering PHB expression (Fig. 3C,D), but did not prevent the protective effects of DPTA in OGD (Fig. 3E). These data indicate that NO-mediated PHB functional modulation does not depend on soluble guanylyl cyclase (sGC) signaling and suggest that protein S-nitrosylation may be involved instead. 
PHB

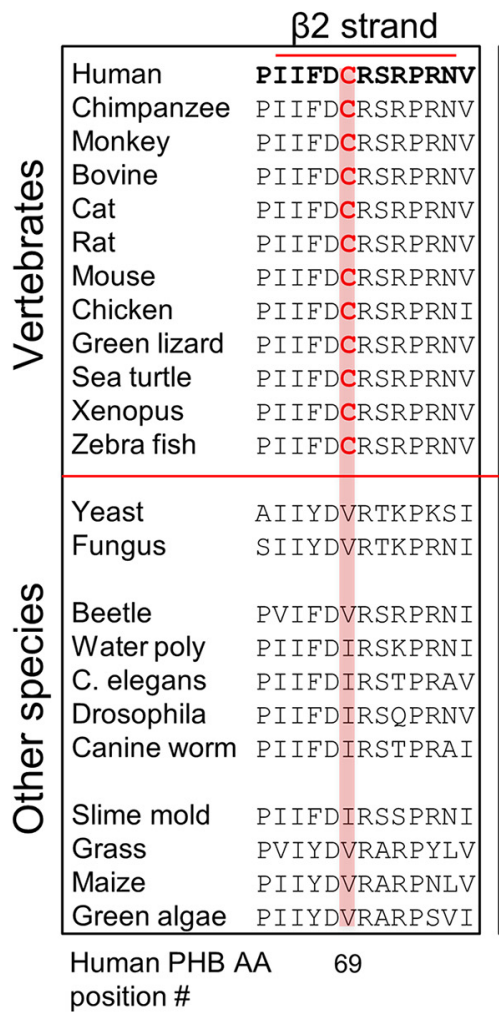

\section{PHB2}

B4 strand

$\alpha 2$ coil

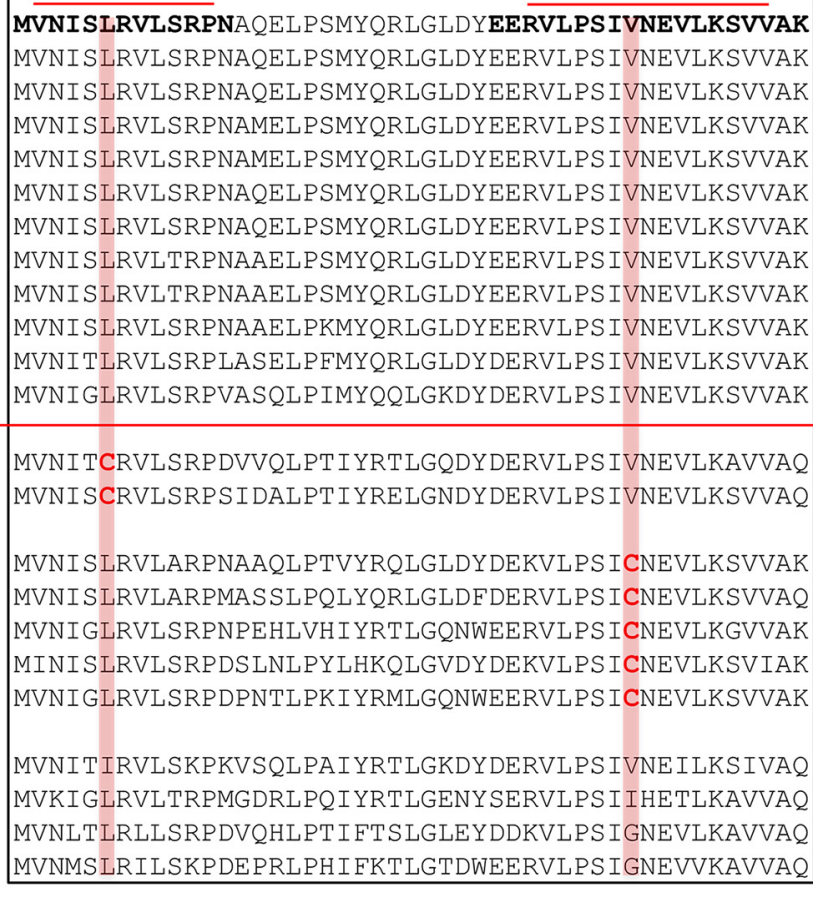

106

137

Figure 4. Conserved Cys residue in PHB and PHB2 protein sequences from different species revealed by alignment. Aligned PHB (left panels) and PHB2 (right panels) sequences were grouped into vertebrate and other species categories. The position Cys occupies is highlighted. $\beta$-Strand and $\alpha$-coil positions previously predicted by a computer generated structure are marked (Winter et al., 2007).
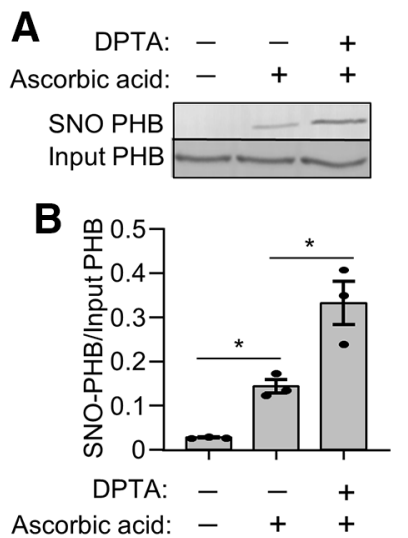
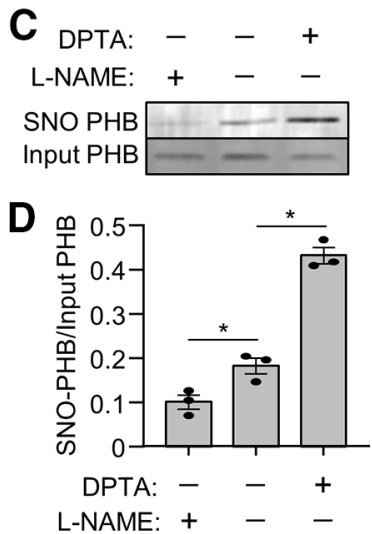

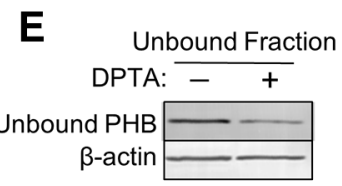

$\mathbf{F}$

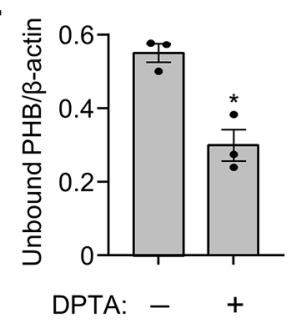

Figure 5. The Cys ${ }^{69}$ residue of PHB is nitrosylated. Neuronal cultures at DIV14 were treated with DPTA (50 $\mu \mathrm{M}$ ) or NOS inhibitor (L-NAME, $100 \mu \mathrm{m}$ ) as indicated, followed by biotin switch assay. $\boldsymbol{A}$, Western blot image showing PHB nitrosylation. At the end of the biotin switch procedure, the nitrosylated proteins collected on avidin beads were subjected to SDS-PAGE and transferred to membranes to detect nitrosylated PHB (SNO-PHB) by Western blot (top). The total PHB content in each sample input was assayed to serve as a loading and normalization control (bottom). $\boldsymbol{B}$, SNO-PHB protein band intensity normalized to the total PHB band intensity of input fractions. ${ }^{*} p<0.05, n=3$ separate experiments. C, Western blot image showing NOS-dependent PHB nitrosylation in neuronal culture. Neurons treated with either NO donor or NOS inhibitor as indicated were lysed for biotin switch assay. SNO-PHB in the bound fraction (top) and total PHB in the input fraction (bottom) as a loading and normalization control were detected by immunoblot. D, SNO-PHB protein band intensity normalized to total PHB content in the input fractions. ${ }^{*} p<0.05, n=3$ /group. $\boldsymbol{E}$, Western blot of unbound (non-nitrosylated) proteins remaining after pulldown of SNO-PHB bound to avidin beads. Proteins in equal volumes of unbound supernatant were separated by SDS-PAGE and probed with specific antibodies for PHB and $\beta$-actin as loading and normalization controls. $\boldsymbol{F}$, Protein band intensity was normalized to $\beta$-actin and expressed as a fold change over control. ${ }^{*} p<0.05, n=3$ separate experiments.

PHB is nitrosylated on its highly conserved unique cysteine $\left(\mathrm{Cys}^{69}\right)$ residue

Protein S-nitrosylation occurs at the thiol group of Cys residues in target proteins. We analyzed $\mathrm{PHB}$ amino acid sequences of various species to determine whether they contain Cys residues that could be nitrosylated. Sequence alignment shows that there is one highly conserved Cys at position $69\left(\mathrm{Cys}^{69}\right)$ in vertebrate species, but not in invertebrates, yeasts, or plants (Fig. 4). On the 

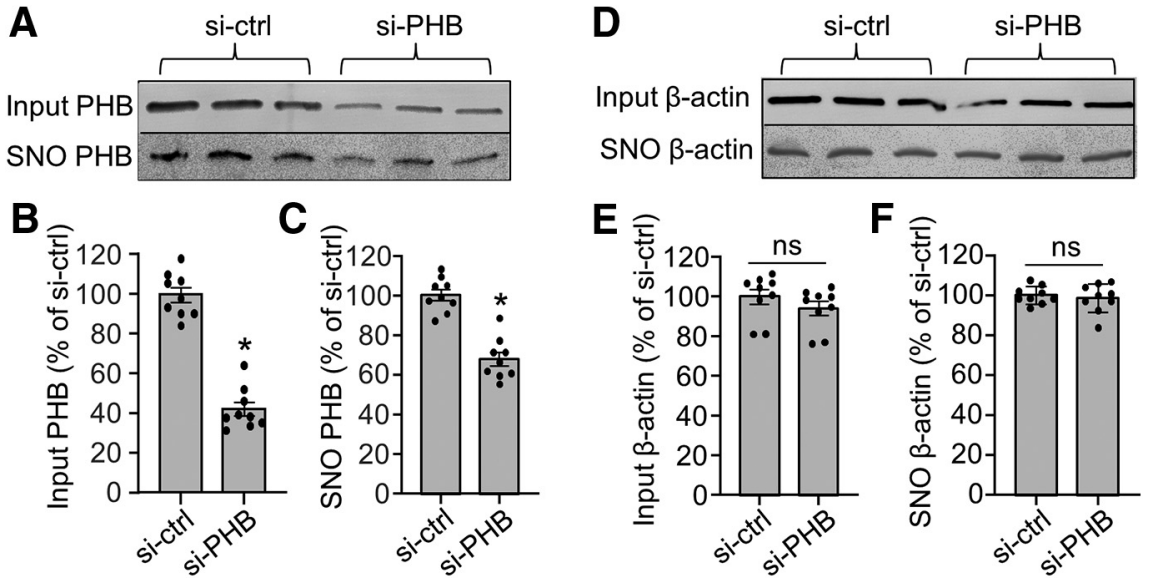

Figure 6. Decrease in endogenous PHB protein level leads to enhanced PHB nitrosylation. DIV7 neuronal cultures were transfected with either si-PHB or si-ctrl for $5 \mathrm{~d}$. The cells were lysed and subjected to the nitrosylation assay by the biotin switch method. $\boldsymbol{A}$, Western blot image showing PHB nitrosylation in endogenous PHB reduced cells (si-PHB) and controls, as indicated. Top, The total PHB content in each sample input assayed to serve as a loading and normalization control. Bottom, The nitrosylated PHB (SNO-PHB). Note the difference in the band intensity between the input and SNO-PHB, indicating elevated nitrosylated fraction of total PHB. B, PHB protein band intensity measurement in input samples. si-PHB-treated samples were normalized to controls and expressed as a percentage of control. ${ }^{*} p<0.05, n=3$ experiments with triplicate of samples per experiment. C, PHB protein band intensity measurement in nitrosylated samples. Band intensity values from si-PHB samples were normalized to si-ctrl and expressed as a percentage of control. $p<0.05, n=3$ experiments with triplicate of samples per experiment. $\boldsymbol{D}$, Western blot image showing $\beta$-actin nitrosylation in si-PHB and control siRNA-treated cells. Top, The total $\beta$-actin content in each sample input assayed to serve as a loading and normalization control. Bottom, The nitrosylated $\beta$-actin (SNO $\beta$-actin). $\boldsymbol{E}, \beta$-Actin protein band intensity measurement in input samples. $\boldsymbol{F}, \beta$-Actin protein band intensity measurement in nitrosylated samples. ns, Not significant. $n=3$ experiments with triplicate of samples per experiment.

other hand, vertebrate PHB2 does not contain a Cys residues, indicating that PHB2 is not susceptible to S-nitrosylation (Fig. 4). Interestingly, in invertebrate animal species the situation is reversed, and there is one conserved Cys residue in PHB2, but not in PHB (Fig. 4, bottom). The high degree of conservation of the single Cys residues in the animal PHB/PHB2 complex suggests that it may play a role in the function of the complex.

We next assessed whether $\mathrm{Cys}^{69}$ of PHB is nitrosylated using a well established biotin switch method (Forrester et al., 2009) and found that PHB is nitrosylated in neurons in basal conditions (Fig. 5A,B). Treatment with L-NAME to inhibit endogenous NO production resulted in reduced PHB nitrosylation by $\sim 45 \%$ (Fig. 5C,D). Conversely, treatment with the NO donor DPTA increased the level of PHB nitrosylation by approximately onefold from baseline (Fig. 5A,B). Omission of ascorbic acid from the biotin-HPDP reaction buffer resulted in weak or undetectable nitrosylated PHB (Fig. 5A,B), demonstrating that PHB contains a bona fide S-nitrosylated cysteine residue (Derakhshan et al., 2007). To further verify the above result, we measured PHB content in the leftover fraction of avidin bead pulldown, which contains all the proteins that are not nitrosylated. The addition of DPTA resulted in significantly less PHB in the unbound (non-nitrosylated) fraction (Fig. 5E,F), confirming that more PHB is nitrosylated in the presence of the NO donor. Together, these data demonstrate that NO leads to PHB S-nitrosylation in a NOS-dependent manner.

\section{Decrease in endogenous PHB protein level leads to enhanced PHB nitrosylation}

It is known that total PHB deletion is lethal to cells but that neurons with partial PHB knockdown remain healthy in normal conditions, without extra stress (Fig. $1 I-K$ ), a phenomenon with an unclear biological basis. Based on the fact that PHB protective function requires the presence of $\mathrm{NO}$ (Fig. 2) and that PHB is nitrosylated at baseline (Fig. 5), we postulated that nitrosylated PHB levels may be altered in neurons with decreased PHB. To test this hypothesis, we assayed PHB nitrosylation in neurons transfected with si-PHB and control siRNA. si-PHB treatment for $5 \mathrm{~d}$ resulted in a significant decrease of PHB protein levels $(42 \pm 5 \%$ of si-ctrl; Fig. $6 A, B)$. Intriguingly, the fraction of nitrosylated PHB in neurons with si-PHB was substantially elevated ( $68 \pm 4 \%$ of si-ctrl; Fig. $6 A, C$ ). On the other hand, the nitrosylation of $\beta$-actin was unchanged (Fig. $6 D-F$ ), suggesting that the elevation of nitrosylated PHB when total PHB is reduced is a specific event. Together with the results shown in Figure 1, the data suggest that increasing PHB nitrosylation could play a compensatory role that support normal cellular activities, when total $\mathrm{PHB}$ is decreased.

\section{Neuronal activity-induced NO modulates PHB S-nitrosylation and neuroprotection}

In order to increase endogenous neuronal NO production, we treated neurons with bicuculline Bic (50 $\mu \mathrm{m}$ for $1 \mathrm{~h})$, a GABA receptor antagonist that promotes neuronal synaptic activity by dampening inhibitory signals (Johnston, 2013). We first assayed NO production in neurons after Bic stimulation with a NO-specific fluorescence dye DAF-FM diacetate (Kojima et al., 1998). NO level was increased in neurons after $1 \mathrm{~h}$ of incubation with Bic, and NO increase was blocked by L-NAME, as expected (Fig. $7 A, B$ ). We next assayed PHB nitrosylation by the biotin switch assay after Bic incubation, and found that Bic induced higher PHB nitrosylation relative to vehicle-treated neurons (Fig. 7C,D). This increase was completely abolished by simultaneous treatment of neurons with Bic and L-NAME (Fig. $7 C, D)$, demonstrating the requirement of NOS for the activitydependent increase of PHB S-nitrosylation. Further, to test whether Bic-induced PHB nitrosylation is associated with neuroprotection, we subjected neurons to Bic treatment followed by OGD. In baseline conditions, Bic did not affect cell viability (Fig. 7E). However, Bic-treated neurons survived OGD better than vehicle controls, while L-NAME abolished the protective effect of Bic (Fig. 7E). Together, these data suggest that neuronal activity regulates NOS-dependent PHB nitrosylation and contributes to neuroprotection. 
A

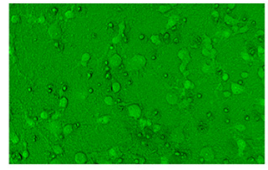

Veh

B

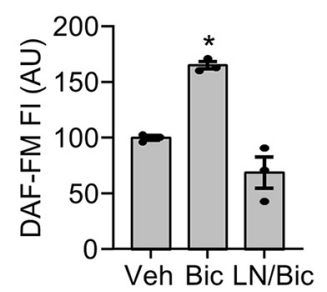

D

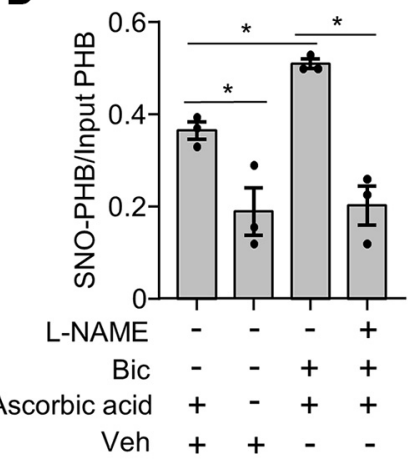

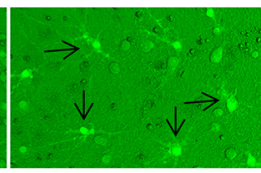

Bic

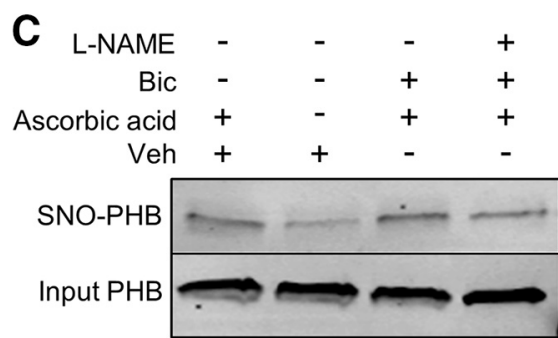

E

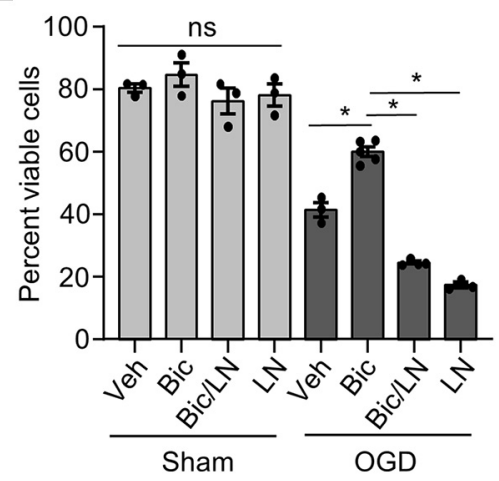

Figure 7. Neuronal cell activity enhances NOS-dependent PHB nitrosylation. Neuronal cultures at DIV14 were pretreated for $6 \mathrm{~h}$ with either L-NAME $(100 \mu \mathrm{m})$ or vehicle followed by Bic $(50 \mu \mathrm{m})$ treatment for $1 \mathrm{~h}$. Cells were then used for the following analyses. $\boldsymbol{A}$, Representative DAF-FM images showing the effect of Bic on intracellular NO levels. After washing off Bic and L-NAME, neurons in 96-well plates were treated with DAF-FM (5 $\mu \mathrm{m})$ for $30 \mathrm{~min}$. After three washes, DAF-FM images were taken using a fluorescence imaging station (ImageXpress Pico). Arrows illustrate the representative Bic-induced DAF-AM fluorescence signal increase in neurons. $\boldsymbol{B}$, Fluorescence intensity measurement of N0 indicator DAF-FM. DAF-FM fluorescence intensity was measured in ImageJ. Values shown were averages from three separate experiments, with $>200$ cells counted each time. ${ }^{*} p<0.05$ compared with other treatments. $C$, Western blot of samples from biotin switch assay demonstrating an increase in nitrosylated PHB (SNO-PHB) after Bic treatment. Total PHB content in input fractions was used as a loading control. Ascorbic acid was omitted in the biotin-HPDP reaction of control samples to serve as a negative control in the biotin switch method. D, Protein band intensity quantification. SNO-PHB band intensity was normalized to the total PHB in the input fractions and expressed as relative protein levels in arbitrary units. ${ }^{*} p<0.05, n=3$ /group. $\boldsymbol{E}$, Neuronal cell viability measurement after Bic treatment. After indicated treatments, neurons were subjected to $O G D$ and cell viability was assessed by cell morphology criteria. ${ }^{*} p<0.05$ compared with all other conditions in $0 G D$ group. There is no statistically significant difference in the sham group.

\section{Discussion}

PHB plays significant roles in diverse cellular processes (ArtalSanz et al., 2003; Merkwirth and Langer, 2009; Merkwirth et al., 2012) or in pathologic conditions such as cancer, inflammation, obesity, and diabetes (Wang et al., 2002; Theiss et al., 2007; Ande et al., 2016a,b; Fan et al., 2017; Mishra and Nyomba, 2017). Work from our laboratory has demonstrated that PHB has a potent neuroprotective role against brain ischemic injury (Zhou et al., 2012; Kurinami et al., 2014; Anderson et al., 2018, 2019; Kahl et al., 2018). However, little is known about how PHB is regulated in neurons.

In this study, we explored the relationship of $\mathrm{NO}, \mathrm{PHB}$, and neuroprotection using an in vitro model of ischemia-reperfusion injury. We show that in order for $\mathrm{PHB}$ to be protective, adequate protein levels are required but not sufficient, as $\mathrm{NO}$ is also required. Thus, $\mathrm{NO}$ and $\mathrm{PHB}$ are mutually interacting for neuronal survival under stress conditions. We further demonstrate that NO modulates $\mathrm{PHB}$ function through protein S- nitrosylation and that this post-translational modification is NOS dependent; and that in neurons $\mathrm{PHB}$ protein decline elevates $\mathrm{PHB}$ nitrosylation, potentially compensating for the partial loss of PHB. Finally, we show that PHB nitrosylation can be stimulated by increased neuronal synaptic activity in a NOS-dependent manner. These findings reveal a previously unrecognized form of functional regulation of $\mathrm{PHB}$ by NO.

In the brain, NO has been established as a neurotransmitter (Garthwaite et al., 1988) and is involved in the control of synaptic functions through modulating neurotransmitter release and plasticity (Brenman and Bredt, 1997; Taqatqeh et al., 2009; Neitz et al., 2011, 2014). In many cases, NO exerts its functions by binding to and activating sGC, which generates the multifunctional second messenger cGMP. For example, NO-mediated cGMP is involved in activity-dependent excitatory synapse development (Nikonenko et al., 2013) and is important for neurite growth and synapse remodeling after axotomy (Cooke et al., 2013). In addition to this classical signaling pathway, NO-mediated post-translational modifications, in particular protein S-nitrosylation, have become increasingly recognized as a form of functional regulation of target proteins (Jaffrey et al., 2001; Hess et al., 2005; Foster et al., 2009). For example, S-nitrosylation of Parkin inhibits its ubiquitin EIII ligase activity and compromises its protective function (Chung et al., 2004). On the other hand, protein S-nitrosylation is essential for normal homeostatic assembly and plasticity of GABAergic synapses (Dejanovic and Schwarz, 2014). In the case of $\mathrm{PHB}$, our results indicate a positive role of $\mathrm{S}$ nitrosylation, since $\mathrm{PHB}$ nitrosylation status dictates $\mathrm{PHB}$ neuroprotective capacity.

In our model, NO executes two separate but related functions leading to neuroprotection against OGD in neurons. One is the enhanced PHB translation to elevate the total $\mathrm{PHB}$ protein levels in neuronal culture that recapitulates what occurs in vivo (Zhou et al., 2012). The other is the direct interaction of $\mathrm{NO}$ with $\mathrm{PHB}$, resulting in the $\mathrm{PHB}$ nitrosylation (Fig. 5). The finding that elevating $\mathrm{PHB}$ protein levels without $\mathrm{NO}$ is not neuroprotective (Fig. 2) adds a layer of regulation in PHB function and underscores a critical role of $\mathrm{NO}$ in cell viability. However, it remains to be elucidated how NO renders PHB functional. One possible mechanisms suggested by our results is through protein nitrosylation. The nitrosylation assay in neurons with reduced PHB supports this hypothesis. Neurons maintain normal viability even when endogenous PHB is reduced by half (Fig. $1 I-K$ ). We found that in neurons with low $\mathrm{PHB}$, the proportion of nitrosylated $\mathrm{PHB}$ is much higher than that in control cells (Fig. 6), possibly compensating for the decrease in total PHB and suggesting that nitrosylation can be dynamically regulated to support cellular activities. In addition, it is possible that NO regulates $\mathrm{PHB}$ function indirectly through nitrosylation of other proteins, which in turn regulates $\mathrm{PHB}$ function. Our results also 
show that $\mathrm{PHB}$ protein levels are critical in this regulation. When endogenous $\mathrm{PHB}$ is decreased, the effect of $\mathrm{NO}$ on neuroprotection is lost (Fig. 1). Therefore, it appears that both PHB and NO are needed to maintain proper neuronal activities. Further work with more specific approaches is needed to fully understand the molecular mechanisms of this $\mathrm{PHB}$ regulation.

$\mathrm{Cys}^{69}$ is within the $\beta 2$ strand and outside of the $\mathrm{PHB} / \mathrm{PHB} 2$ binding interface predicted by an in silico structure model (Winter et al., 2007). Therefore, it is unlikely that PHB nitrosylation status affects its interaction with PHB2. S-Nitrosylation may affect $\mathrm{PHB}$ structure, but it is difficult to hypothesize the precise effect of nitrosylation on $\mathrm{PHB}$ structure and tertiary conformation at this stage, because the structures of $\mathrm{PHB}$ and $\mathrm{PHB} / \mathrm{PHB} 2$ complex have not yet been resolved. Another possible way that S-nitrosylation could affect PHB function is through modulating its binding partners. $\mathrm{PHB}$ has been reported to bind numerous other proteins. For example, PHB interacts with subunits of complex I (Bourges et al., 2004), it forms a complex with histone regulator A, a specific $\mathrm{H} 3.3$ chaperone required for embryonic development, to serve as a key factor in human embryonic stem cell self-renewal (Zhu et al., 2017), and binds to C3 to enhance complement activation (Mishra et al., 2007). In addition, we have previously shown that PHB binds to the essential mitochondrial phospholipid cardiolipin and stabilizes its content, promoting respiratory chain supercomplex assembly and bioenergetic function (Anderson et al., 2018). Thus, it is possible that nitrosylation of $\mathrm{PHB}$ changes its conformation or its ability to interact with other proteins and lipids, allowing it to modulate mitochondrial function.

Proteomic approaches have identified stimuli that induce increases in PHB protein in cells or tissues (Smalla et al., 2008; Bernstein et al., 2012; Poitelon et al., 2015). However, the underlying mechanisms responsible for regulating $\mathrm{PHB}$ content remain unknown. In this study, we demonstrate that the NOmediated increase in $\mathrm{PHB}$ protein does not occur at the transcriptional level as PHB mRNA is unchanged by NO stimulation. Our data support an alternative mechanism in which $\mathrm{PHB}$ is increased by translational activation because inhibition of protein synthesis by CHX abolishes the NO-stimulated increase in $\mathrm{PHB}$. These data suggest a translational regulatory mechanism governing $\mathrm{PHB}$ function in various stress conditions, but further biochemical studies are needed to dissect the exact mechanism by which NO stimulates PHB synthesis from the existing pool of mRNA.

Another interesting observation is that, depending on the species, a conserved unique cysteine residue is present either in $\mathrm{PHB}$ or PHB2, but not in both (Fig. 4). Thus, covalent linkage between $\mathrm{PHB}$ and $\mathrm{PHB} 2$ through a disulfide bridge formation cannot occur. Since both PHB and PHB2 are evolutionarily highly conserved proteins, "unfit" mutations leading to a second Cys residue and a potential disulfide bridge formation in $\mathrm{PHB} / \mathrm{PHB} 2$ dimer may be harmful and eliminated. The one cysteine per PHB/PHB2 dimer configuration could carry evolutionary significance and mediate important interactions of the PHB/PHB2 complex.

In summary, we show here a previously unrecognized direct interaction between $\mathrm{NO}$ and $\mathrm{PHB}$ in NO-mediated neuroprotection, which results in PHB S-nitrosylation modulated by endogenous NOS activity. These findings reveal a novel regulatory mechanism of $\mathrm{PHB}$, a mitochondrial protein critical for maintaining oxidative phosphorylation in conditions of cellular stress (Anderson et al., 2018). However, more work is needed to understand the role of PHB nitrosylation in neuronal brain physiology and in disease conditions.

\section{References}

Ande SR, Nguyen KH, Grégoire Nyomba BL, Mishra S (2016a) Prohibitininduced, obesity-associated insulin resistance and accompanying lowgrade inflammation causes NASH and HCC. Sci Rep 6:23608.

Ande SR, Nguyen KH, Grégoire Nyomba BL, Mishra S (2016b) Prohibitin in adipose and immune functions. Trends Endocrinol Metab 27:531-541.

Anderson CJ, Kahl A, Qian L, Stepanova A, Starkov A, Manfredi G, Iadecola C, Zhou P (2018) Prohibitin is a positive modulator of mitochondrial function in PC12 cells under oxidative stress. J Neurochem 146:235-250.

Anderson CJ, Kahl A, Fruitman H, Qian L, Zhou P, Manfredi G, Iadecola C (2019) Prohibitin levels regulate OMAl activity and turnover in neurons. Cell Death Differ. Advance online publication. Retrieved March 5, 2020. doi: 10.1038/s41418-019-0469-4.

Artal-Sanz M, Tsang WY, Willems EM, Grivell LA, Lemire BD, van der Spek H, Nijtmans LGJ, Sanz MA (2003) The mitochondrial prohibitin complex is essential for embryonic viability and germline function in Caenorhabditis elegans. J Biol Chem 278:32091-32099.

Atochin DN, Clark J, Demchenko IT, Moskowitz MA, Huang PL (2003) Rapid cerebral ischemic preconditioning in mice deficient in endothelial and neuronal nitric oxide synthases. Stroke 34:1299-1303.

Bernstein HG, Smalla KH, Dürrschmidt D, Keilhoff G, Dobrowolny H, Steiner J, Schmitt A, Kreutz MR, Bogerts B (2012) Increased density of prohibitin-immunoreactive oligodendrocytes in the dorsolateral prefrontal white matter of subjects with schizophrenia suggests extraneuronal roles for the protein in the disease. Neuromolecular Med 14:270-280.

Bourges I, Ramus C, Mousson de Camaret B, Beugnot R, Remacle C, Cardol P, Hofhaus G, Issartel JP (2004) Structural organization of mitochondrial human complex I: role of the ND4 and ND5 mitochondria-encoded subunits and interaction with prohibitin. Biochem J 383:491-499.

Brenman JE, Bredt DS (1997) Synaptic signaling by nitric oxide. Curr Opin Neurobiol 7:374-378.

Cho S, Park EM, Zhou P, Frys K, Ross ME, Iadecola C (2005) Obligatory role of inducible nitric oxide synthase in ischemic preconditioning. J Cereb Blood Flow Metab 25:493-501.

Chung KKK, Thomas B, Li X, Pletnikova O, Troncoso JC, Marsh L, Dawson VL, Dawson TM (2004) S-nitrosylation of Parkin regulates ubiquitination and compromises Parkin's protective function. Science 304:1328-1331.

Cooke RM, Mistry R, Challiss RA, Straub VA (2013) Nitric oxide synthesis and cGMP production is important for neurite growth and synapse remodeling after axotomy. J Neurosci 33:5626-5637.

Dejanovic B, Schwarz G (2014) Neuronal nitric oxide synthase-dependent Snitrosylation of gephyrin regulates gephyrin clustering at GABAergic synapses. J Neurosci 34:7763-7768.

Derakhshan B, Wille PC, Gross SS (2007) Unbiased identification of cysteine S-nitrosylation sites on proteins. Nat Protoc 2:1685-1691.

Fan W, Yang H, Liu T, Wang J, Li TW, Mavila N, Tang Y, Yang J, Peng H, Tu J, Annamalai A, Noureddin M, Krishnan A, Gores GJ, MartínezChantar ML, Mato JM, Lu SC (2017) Prohibitin 1 suppresses liver cancer tumorigenesis in mice and human hepatocellular and cholangiocarcinoma cells. Hepatology 65:1249-1266.

Forrester MT, Foster MW, Benhar M, Stamler JS (2009) Detection of protein S-nitrosylation with the biotin-switch technique. Free Radic Biol Med 46:119-126.

Foster MW, Hess DT, Stamler JS (2009) Protein S-nitrosylation in health and disease: a current perspective. Trends Mol Med 15:391-404.

Garthwaite J, Charles SL, Chess-Williams R (1988) Endothelium-derived relaxing factor release on activation of NMDA receptors suggests role as intercellular messenger in the brain. Nature 336:385-388.

Garthwaite J, Southam E, Boulton CL, Nielsen EB, Schmidt K, Mayer B (1995) Potent and selective inhibition of nitric oxide-sensitive guanylyl cyclase by $1 \mathrm{H}-[1,2,4]$ oxadiazolo[4,3-a]quinoxalin-1-one. Mol Pharmacol 48:184-188.

Hess DT, Matsumoto A, Kim SO, Marshall HE, Stamler JS (2005) Protein Snitrosylation: purview and parameters. Nat Rev Mol Cell Biol 6:150-166.

Jaffrey SR, Erdjument-Bromage H, Ferris CD, Tempst P, Snyder SH (2001) Protein S-nitrosylation: a physiological signal for neuronal nitric oxide. Nat Cell Biol 3:193-197. 
Johnston GA (2013) Advantages of an antagonist: bicuculline and other GABA antagonists. Br J Pharmacol 169:328-336.

Kahl A, Anderson CJ, Qian L, Voss H, Manfredi G, Iadecola C, Zhou P (2018) Neuronal expression of the mitochondrial protein prohibitin confers profound neuroprotection in a mouse model of focal cerebral ischemia. J Cereb Blood Flow Metab 38:1010-1020.

Kojima H, Nakatsubo N, Kikuchi K, Kawahara S, Kirino Y, Nagoshi H, Hirata Y, Nagano T (1998) Detection and imaging of nitric oxide with novel fluorescent indicators: diaminofluoresceins. Anal Chem 70:24462453.

Kurinami H, Shimamura M, Ma T, Qian L, Koizumi K, Park L, Klann E, Manfredi G, Iadecola C, Zhou P (2014) Prohibitin viral gene transfer protects hippocampal CA1 neurons from ischemia and ameliorates postischemic hippocampal dysfunction. Stroke 45:1131-1138.

Merkwirth C, Langer T (2009) Prohibitin function within mitochondria: essential roles for cell proliferation and cristae morphogenesis. Biochim Biophys Acta 1793:27-32.

Merkwirth C, Dargazanli S, Tatsuta T, Geimer S, Löwer B, Wunderlich FT, von Kleist-Retzow JC, Waisman A, Westermann B, Langer T (2008) Prohibitins control cell proliferation and apoptosis by regulating OPA1dependent cristae morphogenesis in mitochondria. Genes Dev 22:476488.

Merkwirth C, Martinelli P, Korwitz A, Morbin M, Brönneke HS, Jordan SD, Rugarli EI, Langer T (2012) Loss of prohibitin membrane scaffolds impairs mitochondrial architecture and leads to tau hyperphosphorylation and neurodegeneration. PLoS Genet 8:e1003021.

Mishra S, Nyomba BG (2017) Prohibitin - At the crossroads of obesity-linked diabetes and cancer. Exp Biol Med (Maywood) 242:1170-1177.

Mishra S, Moulik S, Murphy LJ (2007) Prohibitin binds to C3 and enhances complement activation. Mol Immunol 44:1897-1902.

Murad F (1986) Cyclic guanosine monophosphate as a mediator of vasodilation. J Clin Invest 78:1-5.

Murry CE, Jennings RB, Reimer KA (1986) Preconditioning with ischemia: a delay of lethal cell injury in ischemic myocardium. Circulation 74:11241136.

Nakamura T, Lipton SA (2016) Protein S-nitrosylation as a therapeutic target for neurodegenerative diseases. Trends Pharmacol Sci 37:73-84.

Neitz A, Mergia E, Eysel UT, Koesling D, Mittmann T (2011) Presynaptic nitric oxide/cGMP facilitates glutamate release via hyperpolarization-activated cyclic nucleotide-gated channels in the hippocampus. Eur J Neurosci 33:1611-1621.

Neitz A, Mergia E, Imbrosci B, Petrasch-Parwez E, Eysel UT, Koesling D, Mittmann T (2014) Postsynaptic NO/cGMP increases NMDA receptor currents via hyperpolarization-activated cyclic nucleotide-gated channels in the hippocampus. Cereb Cortex 24:1923-1936.

Nijtmans LG, de Jong L, Artal Sanz M, Coates PJ, Berden JA, Back JW, Muijsers AO, van der Spek H, Grivell LA (2000) Prohibitins act as a membrane-bound chaperone for the stabilization of mitochondrial proteins. EMBO J 19:2444-2451.

Nikonenko I, Nikonenko A, Mendez P, Michurina TV, Enikolopov G, Muller D (2013) Nitric oxide mediates local activity-dependent excitatory synapse development. Proc Natl Acad Sci USA 110:E4142-51.
Palmer RM, Ferrige AG, Moncada S (1987) Nitric oxide release accounts for the biological activity of endothelium-derived relaxing factor. Nature 327:524-526.

Park S-E, Xu J, Frolova A, Liao L, O’Malley BW, Katzenellenbogen BS (2005) Genetic deletion of the repressor of estrogen receptor activity (REA) enhances the response to estrogen in target tissues in vivo. Mol Cell Biol 25:1989-1999.

Poitelon Y, Bogni S, Matafora V, Della-Flora Nunes G, Hurley E, Ghidinelli M, Katzenellenbogen BS, Taveggia C, Silvestri N, Bachi A, Sannino A, Wrabetz L, Feltri ML (2015) Spatial mapping of juxtacrine axo-glial interactions identifies novel molecules in peripheral myelination. Nat Commun 6:8303.

Russwurm M, Koesling D (2004) Guanylyl cyclase: NO hits its target. Biochem Soc Symp (71):51-63

Smalla KH, Mikhaylova M, Sahin J, Bernstein HG, Bogerts B, Schmitt A, van der Schors R, Smit AB, Li KW, Gundelfinger ED, Kreutz MR (2008) A comparison of the synaptic proteome in human chronic schizophrenia and rat ketamine psychosis suggest that prohibitin is involved in the synaptic pathology of schizophrenia. Mol Psychiatry 13:878-896.

Steglich G, Neupert W, Langer T (1999) Prohibitins regulate membrane protein degradation by the m-AAA protease in mitochondria. Mol Cell Biol 19:3435-3442

Stomberski CT, Hess DT, Stamler JS (2019) Protein S-nitrosylation: determinants of specificity and enzymatic regulation of S-nitrosothiol-based signaling. Antioxid Redox Signal 30:1331-1351.

Taqatqeh F, Mergia E, Neitz A, Eysel UT, Koesling D, Mittmann T (2009) More than a retrograde messenger: nitric oxide needs two cGMP pathways to induce hippocampal long-term potentiation. J Neurosci 29:93449350.

Theiss AL, Idell RD, Srinivasan S, Klapproth JM, Jones DP, Merlin D, Sitaraman SV (2007) Prohibitin protects against oxidative stress in intestinal epithelial cells. FASEB J 21:197-206.

Theiss AL, Vijay-Kumar M, Obertone TS, Jones DP, Hansen JM, Gewirtz AT, Merlin D, Sitaraman SV (2009) Prohibitin is a novel regulator of antioxidant response that attenuates colonic inflammation in mice. Gastroenterology 137:199-208.

Thuaud F, Ribeiro N, Nebigil CG, Désaubry L (2013) Prohibitin ligands in cell death and survival: mode of action and therapeutic potential. Chem Biol 20:316-331.

Wang S, Zhang B, Faller DV (2002) Prohibitin requires Brg-1 and Brm for the repression of E2F and cell growth. EMBO J 21:3019-3028.

Winter A, Kämäräinen O, Hofmann A (2007) Molecular modeling of prohibitin domains. Proteins 68:353-362.

Zhou P, Qian L, Iadecola C (2005) Nitric oxide inhibits caspase activation and apoptotic morphology but does not rescue neuronal death. J Cereb Blood Flow Metab 25:348-357.

Zhou P, Qian L, D’Aurelio M, Cho S, Wang G, Manfredi G, Pickel V, Iadecola C (2012) Prohibitin reduces mitochondrial free radical production and protects brain cells from different injury modalities. J Neurosci 32:583-592.

Zhu Z, Li C, Zeng Y, Ding J, Qu Z, Gu J, Ge L, Tang F, Huang X, Zhou C, Wang P, Zheng D, Jin Y (2017) PHB associates with the HIRA complex to control an epigenetic-metabolic circuit in human ESCs. Cell Stem Cell 20:274-289.e7. 